\title{
From synchronization to multistability in two coupled quadratic maps
}

\author{
R. Carvalho, B. Fernandez ${ }^{\dagger \dagger}$ and R. Vilela Mendes ${ }^{\dagger}$
}

\begin{abstract}
The phenomenology of a system of two coupled quadratic maps is studied both analytically and numerically. Conditions for synchronization are given and the bifurcations of periodic orbits from this regime are identified. In addition, we show that an arbitrarily large number of distinct stable periodic orbits may be obtained when the maps parameter is at the Feigenbaum period-doubling accumulation point. An estimate is given for the coupling strength needed to obtain any given number of stable orbits.
\end{abstract}

\section{Introduction}

A multistable system is one that possesses a large number of coexisting attractors for a fixed set of parameters. There is ample evidence for such phenomena in the natural sciences, with examples coming from neurosciences and neural dynamics [1] - [7], optics [8] [9], chemistry [10] [11] [12], condensed matter [13] and geophysics [14]. Multistability also seems to be an essential complexity-generating mechanism in a large class of agent-based models [15].

In view of this, it is important to identify the dynamical mechanisms leading to multistability and, in particular, to construct simple models where this phenomenon might be under control. The first mathematical result in

*Laboratório de Mecatrónica, DEEC, Instituto Superior Técnico, Av. Rovisco Pais, 1096 Lisboa Codex, Portugal

${ }^{\dagger}$ Grupo de Física Matemática, Complexo Interdisciplinar, Universidade de Lisboa, Av. Gama Pinto 2, 1699 Lisboa Codex, Portugal

${ }^{\ddagger}$ On leave from Centre de Physique Théorique, CNRS, Luminy, Marseille, France 
this direction was obtained by Newhouse [16 [17 [18 who proved that, near a homoclinic tangency, a class of diffeomorphisms in a two-dimensional manifold has infinitely many attracting periodic orbits (sinks), a result that was later extended to higher dimensions [19]. It has also been proved [20] that, in addition to infinitely many sinks, infinitely many strange attractors exist near the homoclinic tangencies. The stability of the phenomena under small random perturbations has been studied [21] 222].

A second dynamical mechanism leading to multistability is the addition of small dissipative perturbations to conservative systems. Conservative systems have a large number of coexisting invariant sets, namely periodic orbits, invariant tori and cantori. By adding a small amount of dissipation to a conservative system one finds that some of the invariant sets become attractors. Not all invariant sets of the conservative system will survive when the dissipation is added. However, for sufficiently small dissipation, many attractors (mainly periodic orbits) have been observed in typical systems [23] [24] [25]. The problem of migration between attractors and their stability in multipleattractor systems has also been studied by other authors [26] 27]. Most of results are based on numerical evidence. However, using the techniques of deformation stability [28] [29] [30] [31] some rigorous mathematical results 32] may be obtained.

Finally, it has been found recently [33] that, for parameter values near the Feigenbaum period-doubling accumulation point, quadratic maps coupled by convex coupling may have a large number of stable periodic orbits. This is one of the phenomena we study in detail in this paper. The emphasis on quadratic maps near the Feigenbaum accumulation point has a motivation close to the idea of control of chaos [34] [35]. The typical situation in control of chaos, is that of a strange attractor with an infinite number of embedded periodic orbits, all of them unstable. These orbits are then stabilized by several methods. If, instead of a large number of unstable periodic orbits, one has, for example, a large number of sinks, the controlling situation would seem more promising and robust, because the control need not be so accurate. It would suffice to keep the system inside the desired basin of attraction. At the period-doubling accumulation point the Feigenbaum attractor, because of the properties of the flip bifurcations, coexists with an infinite set of unstable periodic orbits. By coupling, as we will show, an arbitrarily large number of orbits may become stable.

The existence of a large number of stable periodic orbits for just two coupled quadratic maps, provides a simple model where multistability is well 
under control, in the sense that not only the nature of the phenomenon is completely understood as one may also compute the range of parameters that provides any desired number of stable orbits. This should be contrasted, for example, with concrete models for the Newhouse phenomenon [36].

Rather than merely focusing on multistability, we also study the phenomenology of two coupled quadratic maps, in particular the bifurcations of periodic orbits and the regime of synchronization.. The stabilization of orbits in the coupled system is similar to that obtained in higher dimensional coupled map lattices [37] with the exception that, due to the restricted dimension of the phase space, the types of bifurcations are different in our system. The results concerning the multistability phenomenon at $\mu=\mu_{\infty}$ also considerably extend, and also correct, some imprecise statements in [33].

\section{Coupled quadratic maps}

Coupled map lattices (CML) are discrete dynamical systems generated by the composition of a local nonlinearity and a coupling. The phase space of the CML considered in this letter is the square $[-1,1]^{2}$ and the dynamics is generated by the map $F_{\epsilon}$ defined as follows. Given a point $(x, y) \in \mathcal{M}$, its image by $F_{\epsilon}$, denoted $(\bar{x}, \bar{y})$ is given by

$$
\left\{\begin{array}{l}
\bar{x}=(1-\epsilon) f(x)+\epsilon f(y) \\
\bar{y}=(1-\epsilon) f(y)+\epsilon f(x)
\end{array}\right.
$$

where $0 \leq \epsilon \leq \frac{1}{2}, f(x)=1-\mu x^{2}$ and $0<\mu<2$.

The map $f$ maps $[-1,1]$ into itself. Therefore, the convex combination in (11) ensures that $F_{\epsilon}\left([-1,1]^{2}\right) \subset[-1,1]^{2}$ and the dynamics is well-defined. We denote the orbit issued from the initial condition $(x, y)$ by the sequence $\left\{\left(x^{t}, y^{t}\right)\right\}_{t \in \mathbb{N}}$, that is to say, $\left(x^{0}, y^{0}\right)=(x, y)$ and $\left(x^{t+1}, y^{t+1}\right)=F_{\epsilon}\left(x^{t}, y^{t}\right)$ for all $t \in \mathbb{N}$.

For the sake of simplicity, we will often employ the variables $s=\frac{x+y}{2}$ and $d=\frac{x-y}{2}$. The previous notation of orbits also applies to these variables for which relation (11) becomes

$$
\left\{\begin{array}{l}
\bar{s}=1-\mu^{2}\left(s^{2}+d^{2}\right) \\
\bar{d}=-\alpha s d
\end{array}\right.
$$

where $\alpha=2 \mu(1-2 \epsilon)$.

Finally, note that the dynamics commutes with the symmetry $\sigma(s, d)=$ $(s,-d)$ or $\sigma(x, y)=(y, x)$ in the original variables. 


\section{Synchronization}

If $d^{t}=0$, then $d^{t+1}=0$ and $s^{t+1}=f\left(s^{t}\right)$. In this case, the orbit is said to be synchronized (from $t$ on). More generally, an orbit is said to synchronize if $\lim _{t \rightarrow \infty} d^{t}=0$ and if all orbits synchronize, then we say to have synchronization of the map. Synchronization is the simplest dynamical regime exhibited by two-dimensional CML.

To determine a sufficient condition for synchronization in our system, we note that for any orbit, one has $\left|s^{t}\right| \leq 1$ for all $t \in \mathbb{N}$. It follows from (2) that the condition $|\alpha|<1$ ensures an exponential decay of $\left|d^{t}\right|$, and hence synchronization.. Since $\alpha \geq 0$, the condition $|\alpha|<1$ is equivalent to the following ones (see Figure 1).

$$
\epsilon=\frac{1}{2} \quad \text { or } \quad \epsilon<\frac{1}{2} \quad \text { and } \quad \mu<\mu_{1}(\epsilon):=\frac{1}{2(1-2 \epsilon)} .
$$

From now on, we assume that $\epsilon<\frac{1}{2}$. The condition $\mu<\mu_{1}(\epsilon)$ is not necessary. Indeed, if for instance $\mu<\frac{3}{4}$, then $f$ has an attracting fixed point in $[-1,1]$, and one can prove that synchronization occurs. When $\epsilon$ is sufficiently small, this happens even though $\mu \geq \mu_{1}(\epsilon)$.

\section{Non-synchronized period-2 orbits}

Starting from synchronization and modifying the parameters, non-synchronized (periodic) orbits appear from bifurcations of synchronized (periodic) ones. To understand this phenomenon, as well as the bifurcations of subsequent orbits, we now study analytically the periodic orbits of period 1 and 2 . Let $\left(s^{*}, 0\right)$ be the synchronized fixed point denoted in $(s, d)$-variables. It exists for any values of the parameters and $F_{\epsilon}$ has no other fixed point in $[-1,1]^{2}$. In $(s, d)$ variables, the Jacobian of $F_{\epsilon}$ at this fixed point is diagonal. One eigenvalue is $f^{\prime}\left(s^{*}\right)$ and the corresponding eigendirection is the diagonal $d=0$. The other eigenvalue is $(1-2 \epsilon) f^{\prime}\left(s^{*}\right)$ and the corresponding direction, orthogonal to the diagonal is referred as the anti-diagonal.

The condition $f^{\prime}\left(s^{*}\right)=-1$ then determines a period-doubling bifurcation, which is of co-dimension 1 if $\epsilon>0$. This is the well-known period-doubling bifurcation of $f$ which creates a synchronized period-2 orbit of $F_{\epsilon}$.

Moreover, one checks that the derivative $f^{\prime}\left(s^{*}\right)$ is negative for any $\mu$. 
Hence the conditions $\epsilon>0$ and $(1-2 \epsilon) f^{\prime}\left(s^{*}\right)=-1$, i.e.

$$
\epsilon>0 \quad \text { and } \quad \mu=\mu_{2}(\epsilon):=\frac{3-4 \epsilon}{4(1-2 \epsilon)^{2}},
$$

determine another co-dimension 1 period-doubling bifurcation of the synchronized fixed point. Indeed the conditions of the corresponding bifurcation theorem (see e.g. [38]) are satisfied when the curve $\mu=\mu_{2}(\epsilon)$ is crossed upward.

The period-2 orbit created at this bifurcation is non-synchronized and symmetric. To show this, denote by $\left(s_{1}, d_{1}\right)$ and $\left(s_{2}, d_{2}\right)$ its components. Since the multiplier $(1-2 \epsilon) f^{\prime}\left(s^{*}\right)$ is negative and the bifurcating direction is the anti-diagonal, we have $d_{1} d_{2}<0$ (sufficiently close to the bifurcation). Because of the $\sigma$ symmetry, the map $F_{\epsilon}$ also has a period-2 orbit with components $\left(s_{1},-d_{1}\right)$ and $\left(s_{2},-d_{2}\right)$. Consequently if $\sigma\left(s_{1}, d_{1}\right) \neq\left(s_{2}, d_{2}\right)$, the system would have two periodic orbits created by a co-dimension 1 bifurcation. This is impossible by the unicity in the bifurcation theorem. Therefore, sufficiently close to the bifurcation, we have $\sigma\left(s_{1}, d_{1}\right)=\left(s_{2}, d_{2}\right)$ and $d_{1} \neq 0$ which is the desired conclusion.

By continuity in the parameters of $F_{\epsilon}$, sufficiently close to the bifurcation, this symmetric orbit is stable with respect to perturbations in one direction (the anti-diagonal direction at the bifurcation) and since $f^{\prime}\left(s^{*}\right)<$ $(1-2 \epsilon) f^{\prime}\left(s^{*}\right)<-1$, it is unstable in the direction orthogonal to the latter.

The bifurcations will now be computed. The orbit with $\sigma\left(s_{1}, d_{1}\right)=$ $\left(s_{2}, d_{2}\right)$ and $d_{1} \neq 0$ exists for any $\mu>\mu_{2}(\epsilon)$ and is the unique (up to time translations) period-2 non-synchronized symmetric orbit of $F_{\epsilon}$ in $[-1,1]^{2}$.

Computing the corresponding Jacobian, one obtains the equation for the multipliers

$$
\lambda^{2}-2\left[(1-2 \varepsilon)\left(S^{2}-D^{2}\right)+2 \varepsilon^{2} S^{2}\right] \lambda+(1-2 \varepsilon)^{2}\left(S^{2}-D^{2}\right)^{2}=0
$$

where

$$
\left\{\begin{array}{l}
S=2 \mu s_{1}=\frac{1}{1-2 \varepsilon} \\
D=2 \mu d_{1}=\frac{\sqrt{4 \mu(1-2 \varepsilon)^{2}-(3-4 \varepsilon)}}{1-2 \varepsilon}
\end{array}\right.
$$

Direct calculations show that, if $\epsilon>0$, the multipliers, say $\lambda_{-}$and $\lambda_{+}$, have zero imaginary part iff $\mu \leq \frac{(2-3 \varepsilon)^{2}}{4(1-2 \varepsilon)^{3}}$. Under this condition, we have $0<\lambda_{-}<1$ if $\mu>\mu_{2}(\epsilon)$ and $\lambda_{+}>1$ iff $\mu_{2}(\epsilon)<\mu<\mu_{3}(\epsilon):=\frac{3}{4(1-2 \epsilon)^{2}}$. (The inequality $\mu_{2}(\epsilon)<\mu_{3}(\epsilon)$ indeed holds if $0<\mu<2$ and $0<\epsilon<\frac{1}{2}$, see Figure 1.) 
Consequently, by increasing $\mu$, the symmetric orbit suffers an inverse pitchfork bifurcation at $\mu=\mu_{3}(\varepsilon)$. This bifurcation is generic for a symmetric orbit in a system with symmetry [38] and the conditions of the bifurcation theorem hold when the curve $\mu=\mu_{3}(\varepsilon)$ is crossed upward.

This bifurcation creates two non-symmetric period-2 orbits (one orbit and its symmetric). We have checked that these orbits exist for any $\epsilon \geq 0$ and $\mu>\mu_{3}(\epsilon)$. For $\epsilon=0$, their components are combinations of a fixed point of $f$ and the components of a period- 2 orbit.

When the imaginary part of $\lambda_{-}$and $\lambda_{+}$is not zero, we have $\left|\lambda_{-}\right|=\left|\lambda_{+}\right|<$ 1 iff $\mu<\mu_{4}(\epsilon):=\frac{5-6 \epsilon}{4(1-2 \epsilon)^{2}}$. (Once again, if $0<\mu<2$ and $0<\epsilon<\frac{1}{2}$, the inequality $\mu_{3}(\epsilon)<\mu_{4}(\epsilon)$ is satisfied, see Figure 1.)

The symmetric orbit is thus stable in the interval $\mu_{2}(\epsilon)<\mu<\mu_{4}(\epsilon)$. If $\epsilon>0$ and the curve $\mu=\mu_{4}(\epsilon)$ is crossed upward, this orbit suffers a Hopf bifurcation creating a locally stable invariant circle. A numerical calculation shows that the latter is destroyed when $\mu$ is sufficiently large or when $\epsilon$ is sufficiently small. Obviously, if $\epsilon=0$, it does not exist and the bifurcation at

$\mu=\mu_{4}(0)=\frac{5}{4}$ which is a period-doubling bifurcation of $f$ creates a period- 4 orbit.

Note that invariant circles in two-dimensional CML resulting from the destabilization of a symmetric orbit and their normal form had already been reported in [39. In that work, the system is also defined by (1), but the local map is $f(x)=a x(1-x)$ and $\epsilon$ may be larger than $\frac{1}{2}$. Figure 2 shows an example of the phenomenology described above. Numerically, it is more convenient to follow the orbits from $\epsilon=0$ to increasing values of the coupling. In this picture, as well as in the following ones the map parameter is $\mu=\mu_{\infty}$ (the accumulation point of the period-doubling cascade). In Figure 2, from $\epsilon=0$ (the circle) to $\epsilon=0.039$ (the point labelled 1), the symmetric orbit is unstable. The figure also shows the invariant circle for $\epsilon=0.03$. Between the points 1 and 2, the symmetric orbit is stable. At point 2, the pitchfork occurs, the symmetric orbit becomes unstable and the non-symmetric orbits are created. Finally, the point $3(\epsilon=0.182)$ corresponds to the collapse on the synchronized fixed point.

\section{The phase opposition orbits}

The previous phenomenology is not restricted to small periods but extend to any power of 2 . In particular, the synchronized period $-2^{p}$ orbit may destabi- 
lize to create a symmetric (non-synchronized) orbit of twice the period.

Given $p \in \mathbb{N}$, let $\left\{s_{i}\right\}_{1 \leq i \leq 2^{p}}$ be the components of the period- $2^{p}$ orbit of $f$. The points $\left\{\left(s_{i}, 0\right)\right\}_{1 \leq i \leq 2^{p}}$ are the components of the synchronized period- $2^{p}$ orbit of $F_{\epsilon}$. By the chain rule and since each Jacobian at $\left(s_{i}, 0\right)$ is diagonal, the corresponding multiplier along the anti-diagonal direction is

$$
(1-2 \epsilon)^{2^{p}} \prod_{i=1}^{2^{p}} f^{\prime}\left(s_{i}\right)
$$

The condition that this multiplier equals -1 determines, if $\epsilon>0$, a codimension 1 period-doubling bifurcation. Applying the reasoning of the previous section to each component $\left(s_{i}, 0\right)$, we conclude that this bifurcation creates an orbit with the property $\sigma\left(s^{t+2^{p}}, d^{t+2^{p}}\right)=\left(s^{t}, d^{t}\right)$ and $d^{t} \neq 0$ for all $t \in \mathbb{N}$, which is called a phase-opposition period- $2^{p+1}$ orbit.

Since $(1-2 \epsilon)^{2^{p}}<1$, this bifurcation occurs only if the bifurcation along the diagonal direction has occurred (the local period-doubling bifurcation of $\left\{s_{i}\right\}_{1 \leq i \leq 2^{p}}$. In other words, the phase opposition period- $2^{p}$ orbit exists only if the synchronized period- $2^{p}$ orbit does. Moreover it follows from Figure 3 that, at least for $\mu=\mu_{\infty}$, the phase opposition period- $2^{p+1}$ orbit exists only if the phase opposition period- $2^{p}$ orbit does. This is confirmed analytically for the period -4 orbit whose existence condition is the instability of the synchronized period-2 orbit in the anti-diagonal direction. One obtains

$$
\mu>\mu_{(4)}(\epsilon)=1+\frac{1}{4(1-2 \epsilon)^{2}},
$$

and $\mu_{2}(\epsilon)<\mu_{(4)}(\epsilon)$ if $0<\epsilon<\frac{1}{4}$ and $\mu_{2}(\epsilon) \geq 2$ and $\mu_{(4)}(\epsilon) \geq 2$ if $\frac{1}{4} \leq \epsilon<\frac{1}{2}$ (see Figure 1).

Furthermore, a numerical calculation at $\mu=\mu_{\infty}$, reported in Figure 3, shows that the succession of bifurcations of a phase opposition orbit does not depend on the period. On this picture, we have plotted the values of $\epsilon$ for the Hopf bifurcation, the pitchfork bifurcation and the period-doubling bifurcation creating the orbit, versus the power of the period. For each period, the phenomenology is identical to that described in the previous section, with an adequate change of scale in $\epsilon$. In addition, the picture shows that several phase opposition orbits may be stable for $\epsilon>0$ fixed. This stabilization is an effect of the coupling that will be discussed below.

Finally, since the phase opposition orbits are the first orbits to appear when the parameters are varied from synchronization and since the first such 
orbit that is created is of period 2, it follows that a necessary and sufficient condition for synchronization is $\mu \leq \mu_{2}(\epsilon)$, the condition for the existence of the latter.

\section{The non-symmetric orbits}

We now analyze the existence and the stability of other period- $2^{p}$ orbits for $\mu=\mu_{\infty}$. Our interest for this value of $\mu$ is that the scaling properties of $f$ are reflected on scaling laws for the periods and values of $\epsilon$ at which the bifurcations occur (see Figure 3 and 8). We only consider the orbits which for $\epsilon=0$ have the same period on projection to both axis $x$ and $y$. These orbits are followed numerically when $\epsilon$ increases and are referred using the phase shift of their components at $\epsilon=0$. For $\mu=\mu_{\infty}$, the map $f$ has a period- $2^{p}$ orbit for each $p \in \mathbb{N}$, whose components for $p$ up to 5 are shown in Figure 5 . In this picture, the numbers reflect the order in which the components are visited and the tree structure represents the origin of each component in the bifurcation cascade. An important notion is the dyadic distance $\delta$ between the components of an orbit. $\delta$ is the number of steps one has to go back in the bifurcation tree to meet a common component.

The dyadic distance is used to characterize the families of periodic orbits that we are considering. For instance, the coordinates of each component of a synchronized orbit are at distance 0 , those of a phase-opposition orbit are at distance 1. Accordingly, when we speak of distance- $k$ orbit we refer to the dyadic distance of the coordinates of its components.

For any $\delta \geq 1$, there are $2^{\delta-1}$ different orbits with distance $\delta$ which have coordinates out of phase by $2^{p-\delta}+\alpha_{1} 2^{p-\delta+1}+\alpha_{2} 2^{p-\delta+2}+\cdots+\alpha_{d-1} 2^{p-1}$ steps, with $\alpha_{i} \in\{0,1\}$. The distance of a period- $2^{p}$ orbit is at most $p(p \in \mathbb{N})$. For $\epsilon=0$, the only symmetric orbits are those at distance 0 and 1 . This property is preserved for $\epsilon>0$ as shows Figure 6 for $\delta=2$. The succession of bifurcations of orbits with distance $\delta \geq 2$ should then differ from those with distance 1. The differences are seen in Figure 6 which shows the evolution of the eigenvalues. For $\epsilon=0$, the orbit is unstable. When $\epsilon$ increases, it suffers two collisions with orbits of twice the period when the eigenvalues cross -1 and then becomes stable. (When decreasing $\epsilon$, these collisions would be period-doubling bifurcations.) If $\epsilon$ increases further, the orbit collides with an unstable one of the same period in a saddle-node bifurcation when the larger eigenvalue reaches 1 . For larger values of $\epsilon$, the orbit does not exist. 
The unstable orbit with which it collides is the one that at $\epsilon=0$ has period $2^{p}$ in one projection and $2^{p-1}$ in the other. For higher dyadic distances, the overall variation of the eigenvalues is similar to the $\delta=2$ case. Figure 7 shows a typical example of these phenomena for the case $\delta=2$. Between $\epsilon=0$ and the point labelled 1 in the figure, the orbit is unstable. The point 1 corresponds to the smaller eigenvalue crossing -1 (see Figure 6). Therefore, between the point 1 and 2 , the orbit is stable. It disappear at the point 2 when it collides with an unstable orbit of the same period.

\section{Multistability}

We have seen that the coupling stabilizes the orbits with distance larger than 0 at $\mu=\mu_{\infty}$. There are indeed two mechanisms responsible for this stabilization.. The determinant of the Jacobian of a period- $2^{p}$ orbit is

$$
(1-2 \epsilon)^{2^{p}}\left(-2 \mu_{\infty}\right)^{2^{p+1}} \prod_{i=1}^{2^{p}} x_{i} y_{i}
$$

The term $(1-2 \epsilon)^{2^{p}}$ coming from the coupling decreases when $p$ increases. However, there is yet a second stabilizing mechanism. Denote by $\Gamma(\epsilon)$ the remaining factor in the determinant

$$
\Gamma(\epsilon)=\left(-2 \mu_{\infty}\right)^{2^{p+1}} \prod_{i=1}^{2^{p}} x_{i} y_{i}
$$

Without coupling, $\Gamma(0)$ is simply the square of the multiplier of $f$ for the periodic orbit. From the properties of the Feigenbaum - Cvitanovic functional equation it follows 33 that this factor converges to a fixed value around -1.6 when $p$ increases. The coupling however, changes the position of the orbit components in such a way that this factor also decreases. It is the combined action of this decrease with the contraction of the coupling that brings the eigenvalues into the interior of the unit circle and stabilizes the orbits.

For small $\epsilon$ there is a simple geometrical interpretation for the variation of $\Gamma(\epsilon)$. The reason why in the one dimensional map the product $\prod_{i=1}^{2^{p}} x_{i}$ remains constant, when $p$ grows, is because each time the period doubles, the doubling in the number of factors greater than one is compensated by the fact that the component of the orbit closest to zero approaches zero a 
little more. For the unstable orbits along the period-doubling chain, the orbit components closest to zero alternate on each side of the origin. The contracting effect of the convex coupling tends to bring the orbits back in the period-doubling hierarchy. Therefore, because the component closest to zero has to move across the origin for the orbit to approach the one with half the period, this implies that the product of the coordinates is going to decrease. The greater the dyadic distance between the orbit projections on the axis, the greater will be the perturbation that the original (one-dimensional) orbits suffer. Therefore one expects the contracting effect in $\Gamma(\epsilon)$ to increase with the dyadic distance. This effect is quite apparent on Figure 8 which shows the stabilizing and destabilizing lines for orbits with distance from 1 to 4 . The shift downwards of the stable regions for successively larger dyadic distances implies that the smaller $\epsilon$ is, the larger the number of distinct stable orbits that are obtained. An accurate numerical estimate of the number of distinct orbits is obtained by computing the derivative $D(p, \delta)=\partial\left(\prod_{i=1}^{2^{p}} x_{i} y_{i}\right) / \partial \epsilon$ at $\epsilon=0$ for each $p$ and dyadic distance $\delta$. Actually this derivative provides an accurate estimate of $\Gamma(\epsilon)$ itself, because this one varies almost linearly with $\epsilon$ for most of the stable range of the orbits. On Figure 9, the scaling properties, when $p$ grows, of this derivative are shown. From these results one computes

$$
\log (-D(p, \delta))=g(\delta)-2^{p+1}
$$

with

$$
g(\delta) \simeq 0.907 \delta+3.475
$$

Notice that in Figure 9 there is more than one data point for each pair $(p, \delta)$ which correspond to non-equivalent orbits with the same dyadic distance. Two other useful estimates are:

- the value of the smallest $\epsilon$ parameter that stabilizes an orbit of dyadic distance $\delta$ equal to the power $p$

$$
\log \epsilon_{\delta=p}^{\min }=-1.535 p-1.547
$$

- the value of the largest $\epsilon$ parameter for which a $\delta=1$ orbit is stable

$$
\log c_{\delta=1}^{\max }=-0.679 p-1.235
$$


From this, one obtains the result that at least $k$ distinct stable orbits are obtained if

$$
0<\epsilon \lesssim \exp (-0.99-1.22 k)
$$

$k$ is only a lower bound on the number of distinct stable periodic orbits, because here we have studied only orbits with the same period under projection in the two axis.

In conclusion: for sufficiently small $\epsilon$ an arbitrarily large number of distinct stable periodic orbits is obtained. However, for any fixed $\epsilon$, it is an arbitrarily large number that is obtained, not an infinite number. Most orbits either synchronize (and are then unstable) or disappear as $\epsilon$ grows. As a result, a reasoning based on the implicit function theorem, as used in [33] is misleading. Given a sequence of orbits of different periods, even if they remain as orbits for a small perturbation, that does not mean that their (smallest) periods remain distinct.

\section{References}

[1] S. Schiff et al.; Nature 370, 615 (1994)

[2] J. Foss et al.; Phys. Rev. Lett. 76, 708 (1996)

[3] H. S. Seung; Neural Networks 11, 1253 (1998)

[4] W. J. Freeman; Biol. Cyb. 56, 139 (1987)

[5] J. A. Dente and R. Vilela Mendes; Phys. Lett A211, 87 (1996)

[6] R. S. MacKay and J. - A. Sepulchre; Physica D 82, 243 (1995)

[7] H. Bohr, P. McGuire, C. Pershing and J. Rafelski; Threshold noise as a source of volatility in random synchronous asymmetric neural networks, Univ. Arizona report, Tucson 1997.

[8] S. M. Hammel et al.; J. Opt. Soc. Am. B2, 552 (1985)

[9] M. Brambilla et al.; Phys. Rev. A43, 5114 (1991)

[10] P. Marmillot et al.; J. Chem . Phys. 95, 1206 (1991)

[11] J. P. Laplante and T. Erneux; Physica A188, 89 (1992) 
[12] K. L. C. Hunt et al.; J. Chem. Phys. 96, 7019 (1992)

[13] F. Prengel et al.; Phys. Rev. B50, 1705 (1994)

[14] S. Yoden; Nonlinear Analysis, Methods and Applications, 30, 4607 (1997)

[15] R. Vilela Mendes; Physica A276, 550 (2000)

[16] S. Newhouse; Proc. A. M. S. Symp. Pure Mat. 14, 191 (1970)

[17] S. Newhouse; Topology 13, 9 (1974)

[18] S. Newhouse; Publ. Math. I.H.E.S. 50, 101 (1979)

[19] J. Palis and M. Viana; Annals of Math. 140, 207 (1994)

[20] E. Colli; Ann. de l' Institut Henri Poincaré (Analyse non-linéaire) 15, 539 (1998).

[21] V. Araújo; Attractors and time averages for random maps, IMPA report F-109/98, to appear in Ann. de l' Institut Henri Poincaré (Analyse nonlinéaire).

[22] V. Araújo; Infinitely many stochastically stable attractors, Univ. Porto report 1999-21.

[23] L. Poon and C. Grebogi; Phys. Rev. Lett. 75, 4023 (1995)

[24] U. Feudel, C. Grebogi, B. R. Hunt and J. A. Yorke; Phys. Rev. E54, 71 (1996)

[25] U. Feudel and C. Grebogi; Chaos 7, 597 (1997)

[26] R. Weigel and E. Atlee Jackson; Int. J. of Bifurcation and Chaos 8, 173 (1998)

[27] K. Kaneko; Phys. Rev. Lett. 78, 2736 (1997)

[28] R. Vilela Mendes and J. T. Duarte; J. Math. Phys.; 24, 1772 (1983)

[29] R. Vilela Mendes and J. T. Duarte; Lett. Math. Phys. 6, 249 (1982)

[30] R. Lima and R. Vilela Mendes; Nonlinear Analysis, 12, 1061 (1988) 
[31] R. Vilela Mendes; in Deformation Theory of Algebras and Structures and Applications , pp. 981 - 1013, M. Hazewinkel and M. Gerstenhaber (Eds.) Kluwer 1988

[32] R. Vilela Mendes; Multistability in dynamical systems, chaodyn/9904004, to appear in Dynamical systems: From crystal to chaos, World Scientific, Singapore 2000.

[33] R. Carvalho, R. Vilela Mendes and J. Seixas; Physica D126, 27 (1999)

[34] E. Ott, C. Grebogi and J. A. Yorke; Phys. Rev. Lett. 64, 1196 (1990)

[35] Focus Issue on Control and Synchronization of Chaos; Chaos 7, no.4 (1997)

[36] J. M. Gambaudo and C. Tresser; J. Stat. Phys. 32, 455 (1983)

[37] R.E. Amritkar and P.M. Gade; Phys. Rev. E 492617 (1994).

[38] J. Guckenheimer and P. Holmes, Nonlinear Oscillations, Dynamical Systems, and Bifurcations of Vector Fields, Springer-Verlag, (1983).

[39] K. Kürten and G. Nicolis; Physica A 245, 446 (1997). 


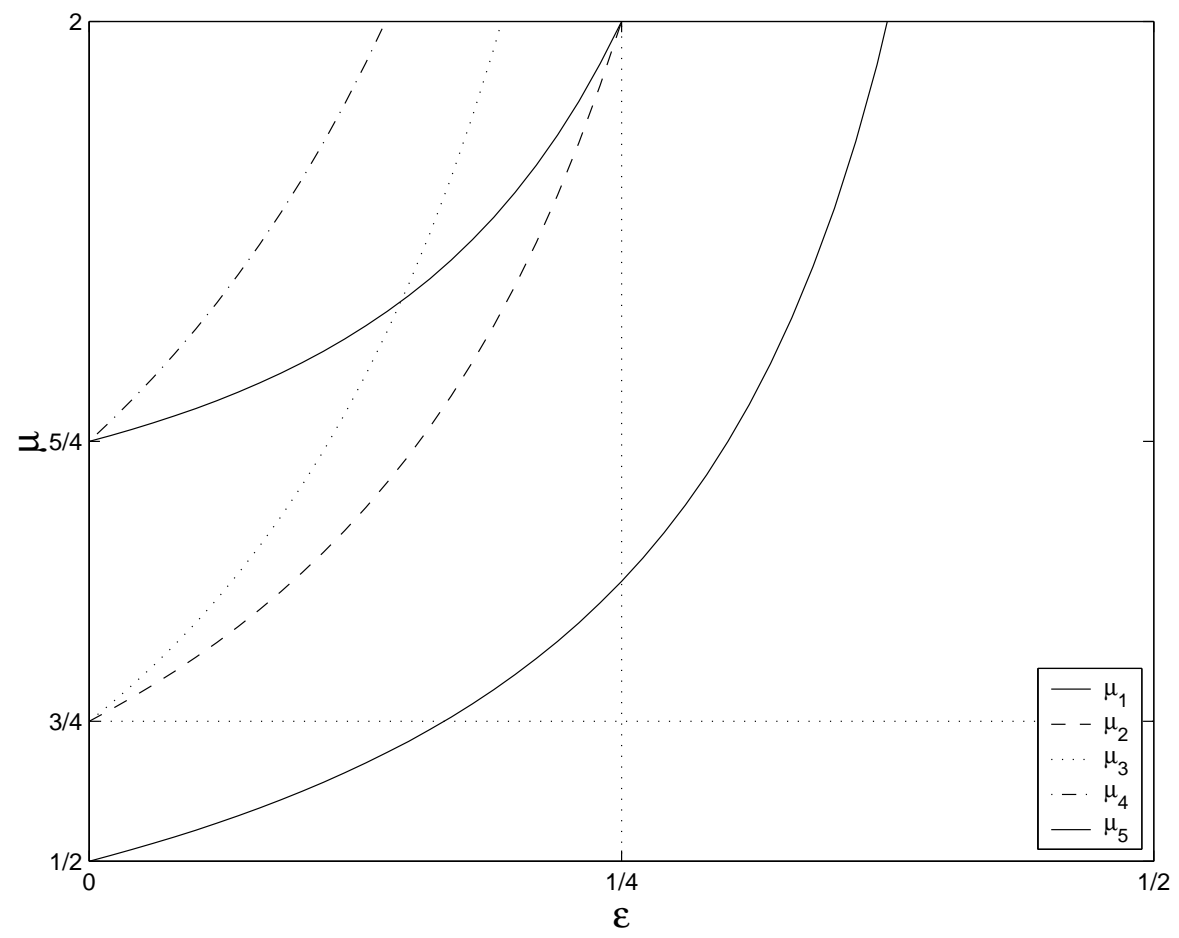

Figure 1: Synchronization curve $\mu=\mu_{1}(\epsilon)$ and other bifurcations curves. $\mu_{2}(\epsilon)$ is the period-doubling of the synchronized fixed point (i.e. birth of the phase opposition period-2 orbit), $\mu_{3}(\epsilon)$ is the pitchfork of the phase opposition period-2 orbit and $\mu_{4}(\epsilon)$ the Hopf bifurcation of the same orbit. $\mu_{(4)}(\epsilon)$ is the period-doubling of the synchronized period-2 orbit (i.e. birth of the phase opposition period-4 orbit) 


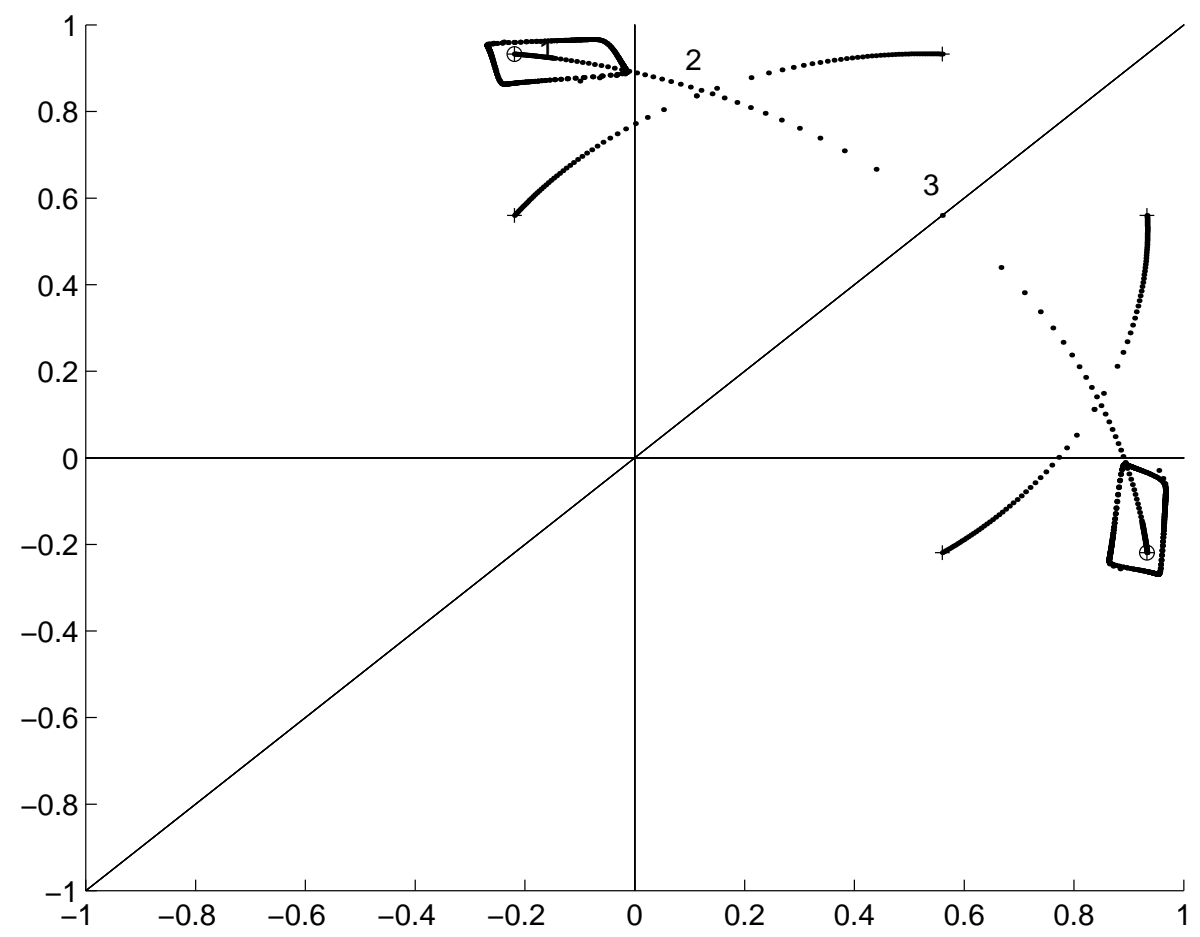

Figure 2: $\varepsilon$-evolution of a period-2 orbit for $\mu=\mu_{\infty}$. An example of invariant circle is also shown for $\epsilon=0.03$ 


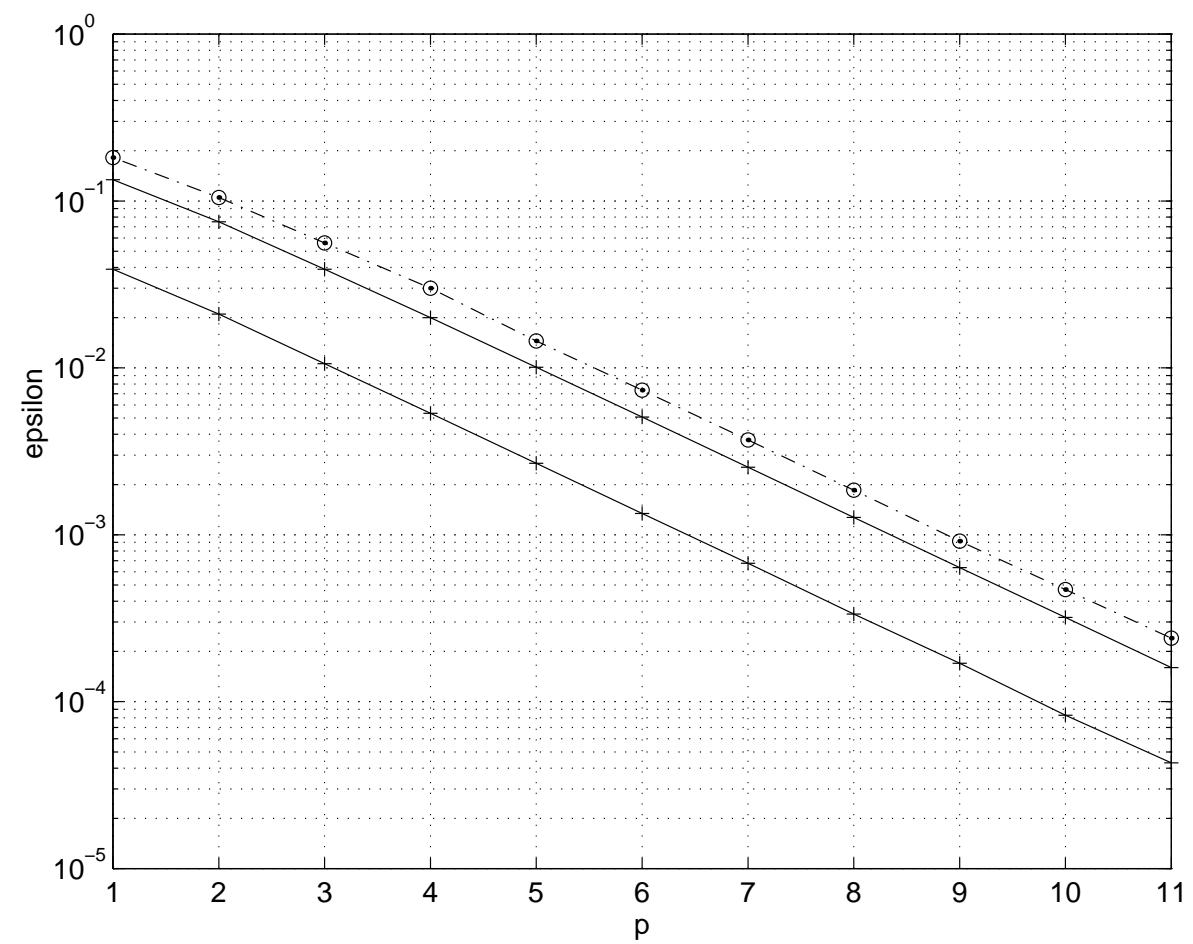

Figure 3: Bifurcation values of $\epsilon$ for the phase opposition orbits. From top to bottom, birth, pitchfork and Hopf bifurcation 

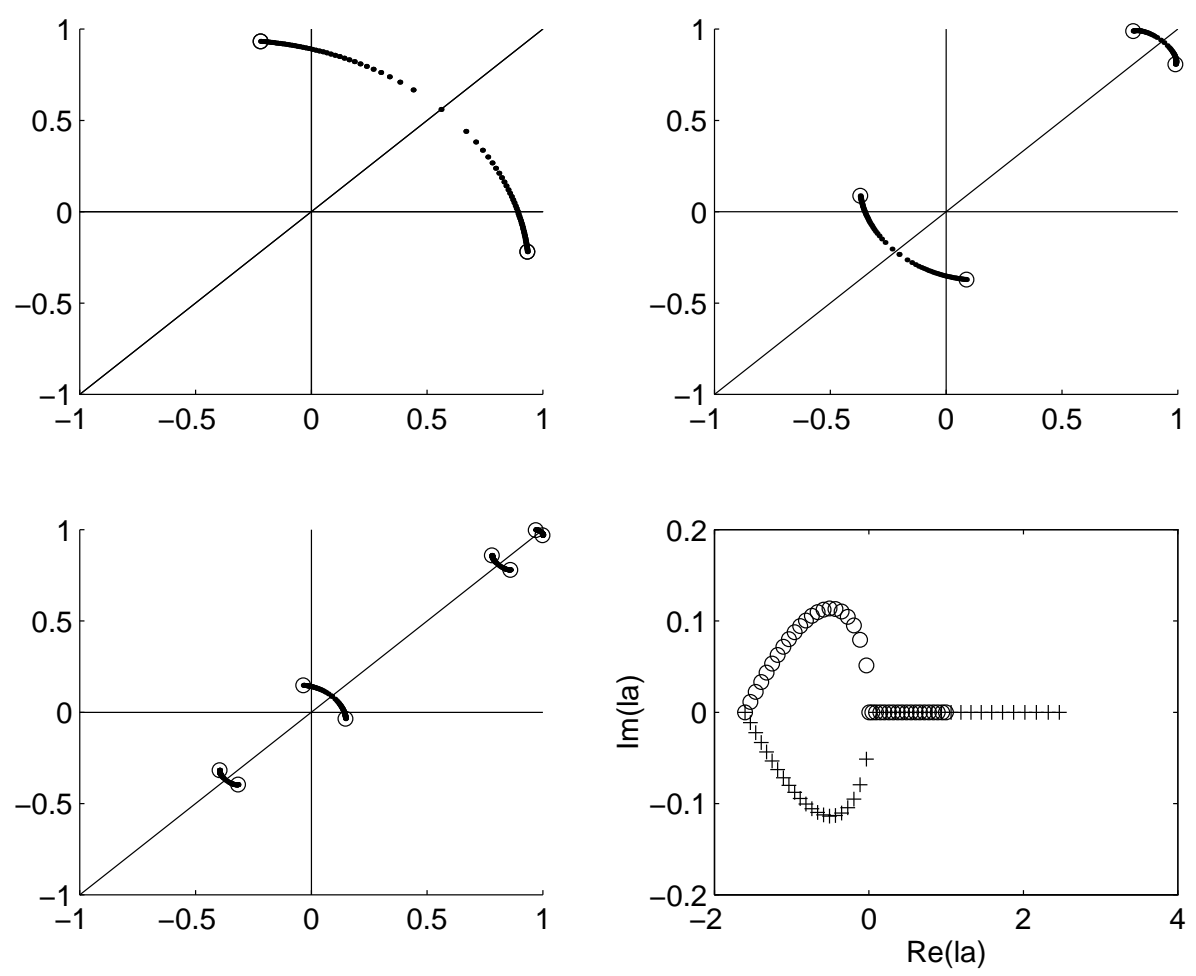

Figure 4: $\varepsilon$-evolution of distance-1 orbits and their multipliers at $\mu=\mu_{\infty}$ 


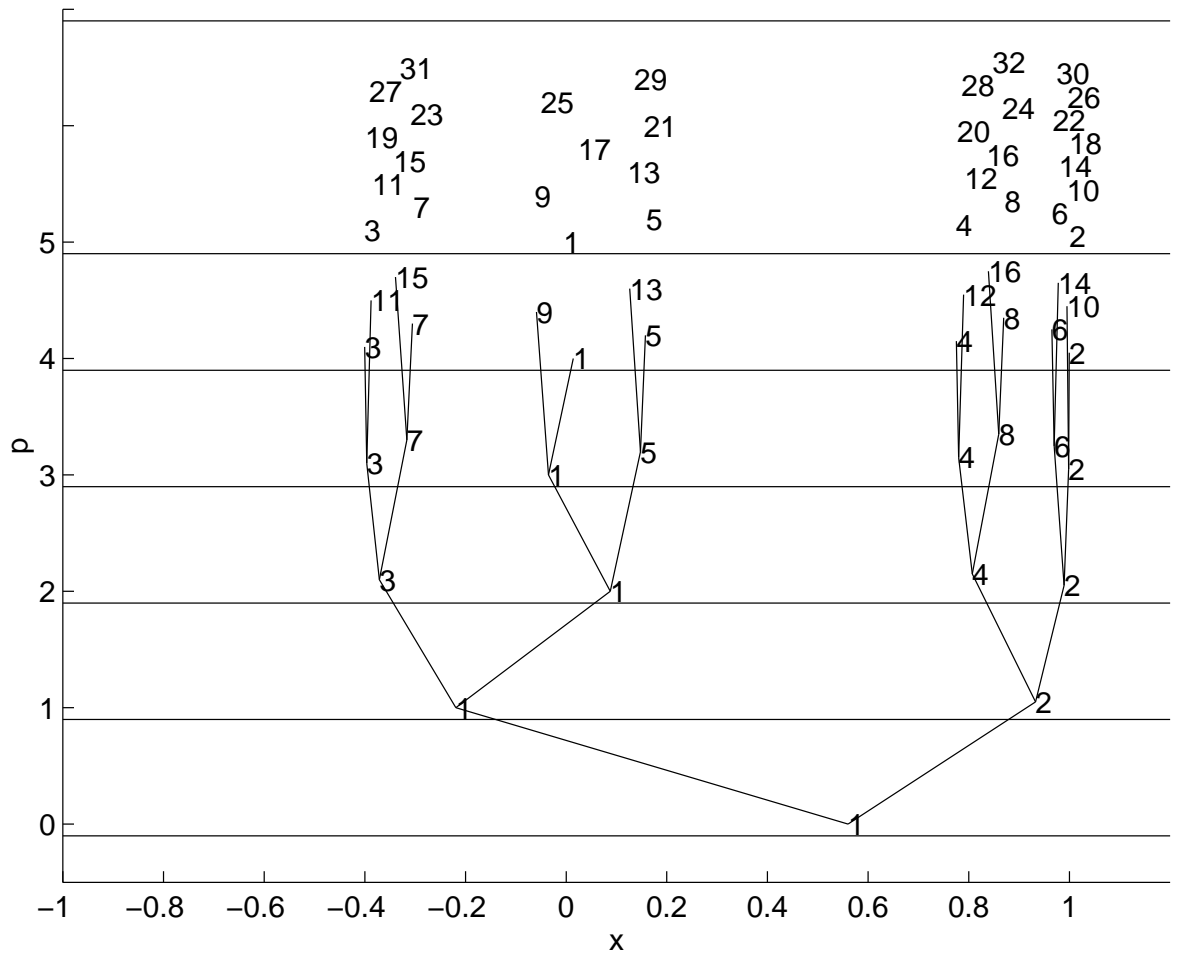

Figure 5: Periodic orbits for the one-dimensional map at $\mu=\mu_{\infty}$ 

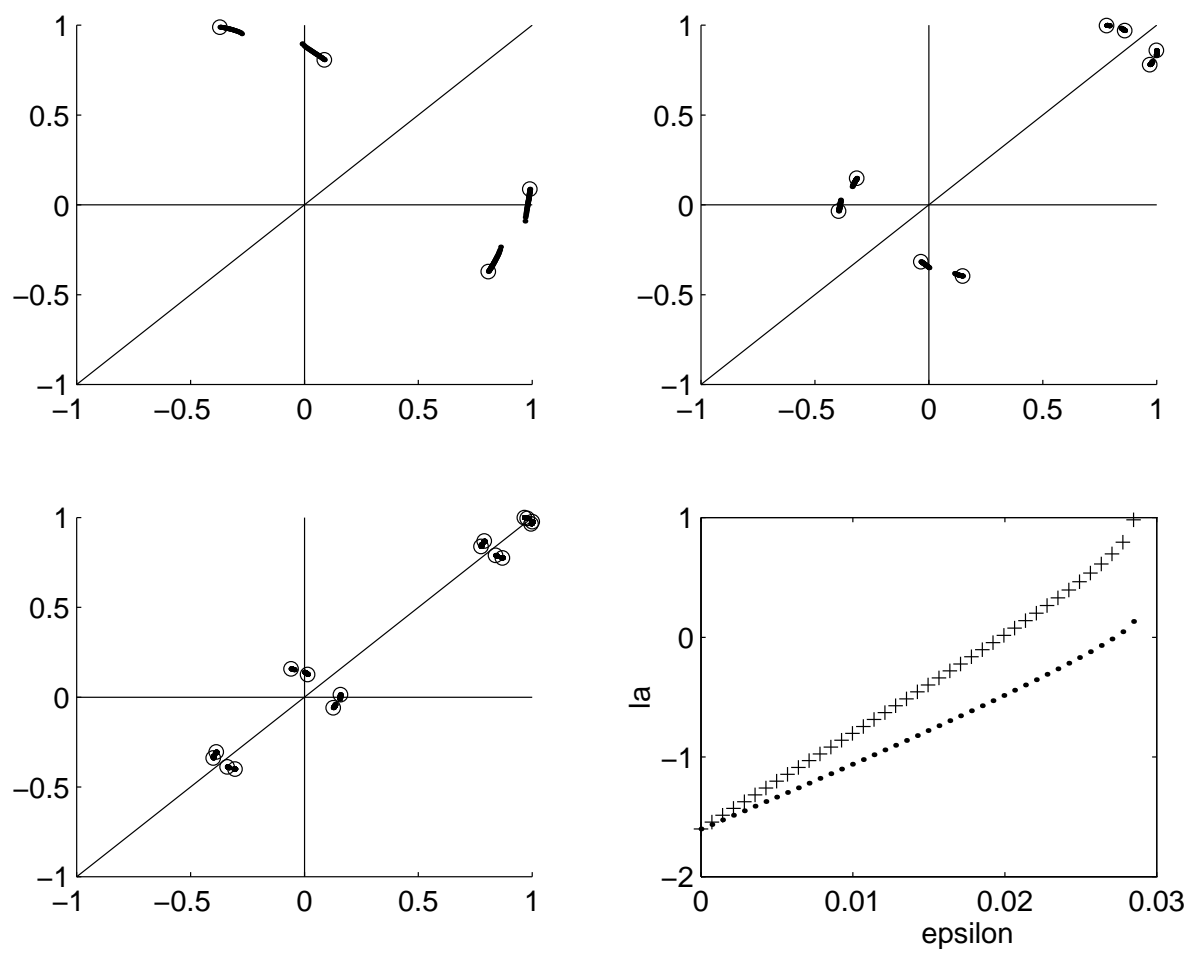

Figure 6: $\varepsilon$-evolution of distance-2 orbits and their multipliers at $\mu=\mu_{\infty}$ 


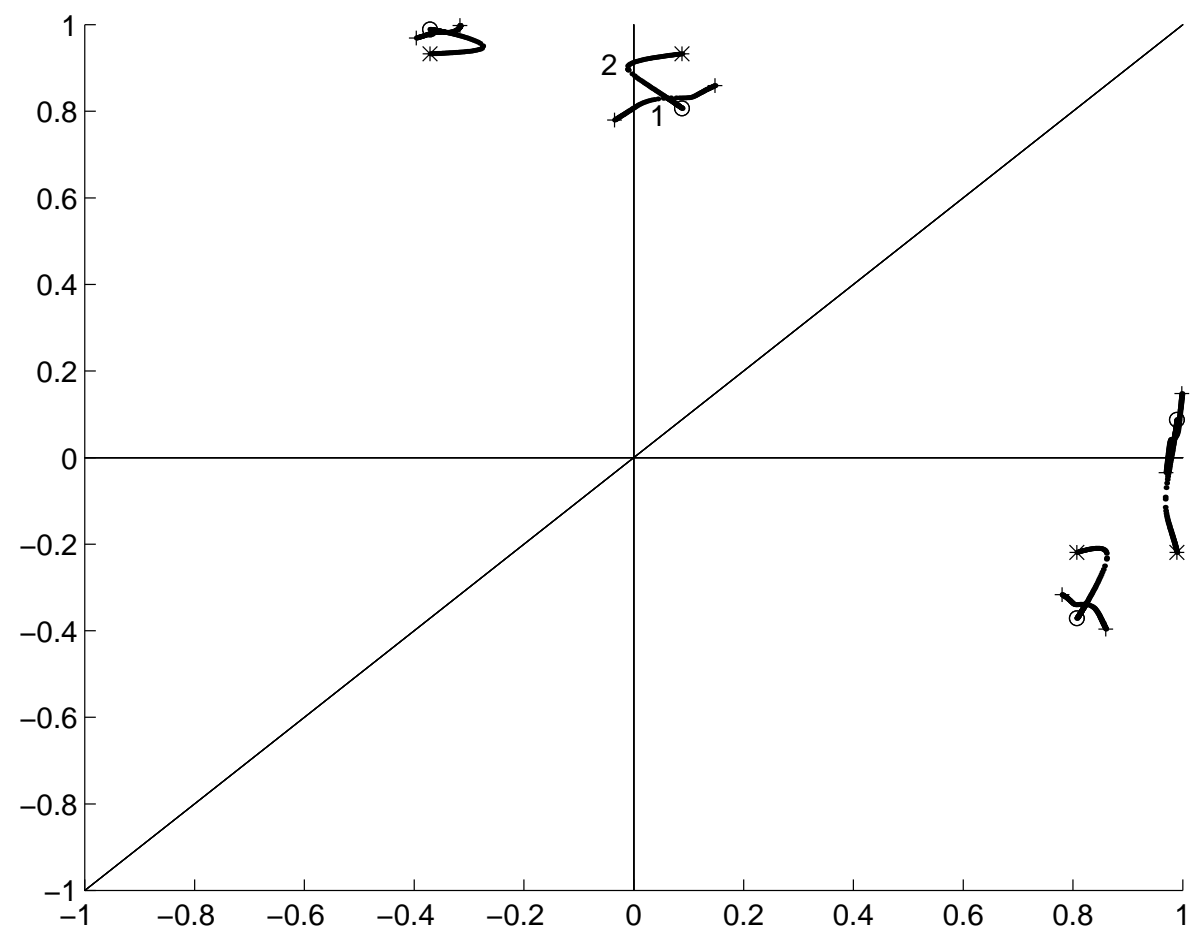

Figure 7: $\varepsilon$-evolution of a period-4 distance-2 orbit and its bifurcating orbits at $\mu=\mu_{\infty}$ 


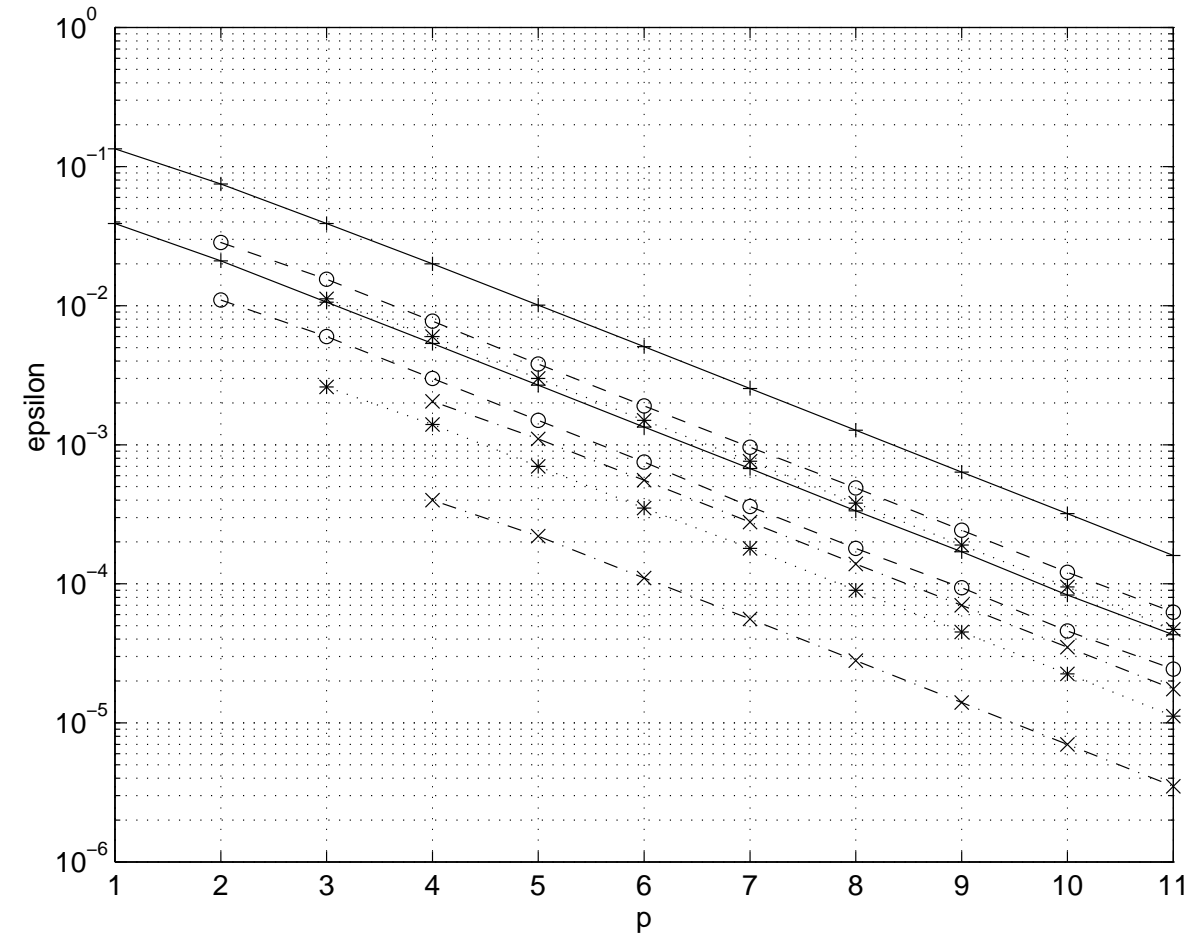

Figure 8: Stability lines of orbits from distance 1 (top) to 4 (bottom) 


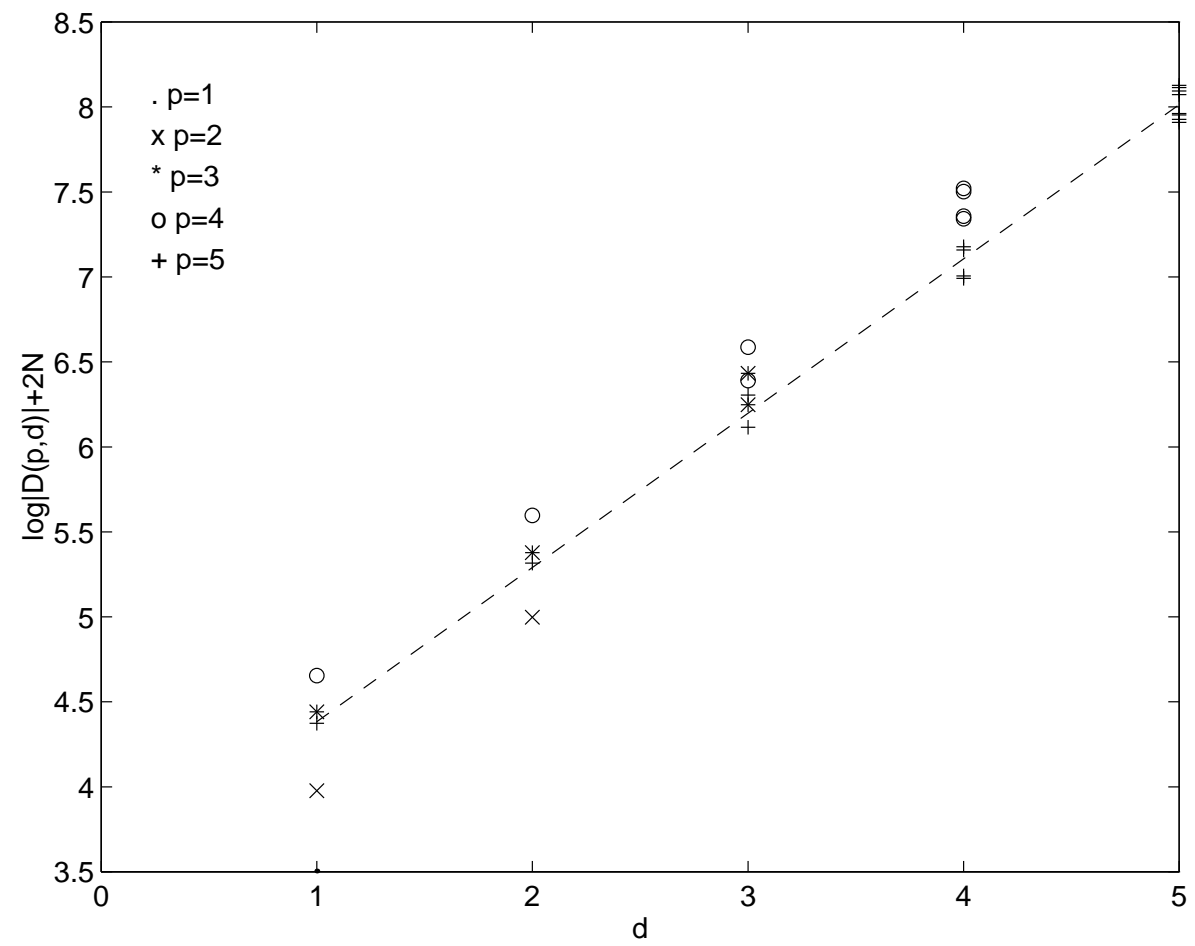

Figure 9: Scaling properties of $D(p, \delta)$ 